\title{
Photoisomerization and Photocyclization of 5-Styryloxazole ${ }^{\dagger}$
}

\author{
Ivana Šagud, ${ }^{a}$ Marija Šindler-Kulyk, ${ }^{a, *}$ Anna Spalletti, ${ }^{\mathrm{b}, *}$ and Ugo Mazzucato ${ }^{\mathrm{b}}$ \\ a Department of Organic Chemistry, Faculty of Chemical Engineering and Technology, University of Zagreb, \\ Marulićev trg 19, HR-10000 Zagreb, Croatia \\ ${ }^{\mathrm{b}}$ Department of Chemistry, Biology and Biotechnologies and Centro di Eccellenza Materiali Innovativi Nanostrutturati \\ (CEMIN), University of Perugia, Piazza Università 1, 06123 Perugia, Italy
}

RECEIVED MARCH 19, 2014; REVISED MAY 6, 2014; ACCEPTED MAY 9, 2014

\begin{abstract}
This paper describes the competition of the relaxation processes (fluorescence, isomerization and cyclization) of 5-styryloxazole in the first excited singlet state. The study is carried out under mild conditions and in the first stage of irradiation to compare the photobehaviour with that reported in a previous work on preparative scale. The quantum yields of the radiative and reactive deactivation are compared with those of the analogous compounds containing pentatomic heteroaryl groups.
\end{abstract}

Keywords: electrocyclization, fluorescence, oxazoles, photochemistry

\section{INTRODUCTION}

The competition between the radiative (fluorescence) and reactive (geometrical isomerization and cyclization) relaxation of stilbene and its analogues has been a subject widely investigated in the last decades as model photoprocesses to understand the dynamics of photoinduced molecular rearrangements. ${ }^{1}$

The $\mathrm{E} \rightarrow \mathrm{Z}$ isomerization occurs generally by the well known diabatic mechanism implying twist around the ethylenic bond towards an energy minimum at the perpendicular configuration (perp), at about $90^{\circ}$, followed by a $\mathrm{S}_{1} \rightarrow \mathrm{S}_{0}$ internal conversion (IC) or $\mathrm{T}_{1} \rightarrow \mathrm{S}_{0}$ intersystem crossing (ISC) and relaxation to the groundstate trans (E) and cis (Z) isomers in roughly a 1:1 branching ratio. The photoreaction can be thus schematised: ${ }^{1,3} \mathrm{E}^{*} \rightarrow{ }^{1,3}$ perp* $\rightarrow{ }^{1}$ perp $\rightarrow \alpha^{1} \mathrm{Z}+(1-\alpha)^{1} \mathrm{E}$, where the partitioning factor $\alpha$ is assumed to be $\sim 0.5$ in most cases. ${ }^{2}$ In some less common cases, specific structures may stabilize the $S_{1}$ state of the E geometry and increase the energy barrier to twisting thus leading to a practically photostable ${ }^{1,3} \mathrm{E}^{*}$ state and a ${ }^{1,3} \mathrm{Z}^{*}$ isomer able to reach the fluorescent $E^{*}$ state on the same potential energy curve (adiabatic mechanism, ${ }^{1,3} \mathrm{Z}^{*} \rightarrow{ }^{1,3} \mathrm{E}^{*}$ ), followed by the relaxation pathways of ${ }^{1,3} \mathrm{E}^{*}$ seen above. $^{3}$

While the cis-trans $(Z-E)$ photoisomerization can occur in both the singlet and triplet states, photocyclization, which starts from the $Z$ isomer produced by pho- toisomerization of the more stable $E$ isomer, is generally accepted to occur in the singlet manifold. In the case of the model molecule stilbene, photocyclization leads to an unstable coloured 4a,4b-dihydrophenanthrene (DHP) which, in the presence of an oxidant, loses the two hydrogen atoms in trans stereochemistry to give the stable polynuclear phenanthrene-type (P) arene. ${ }^{4-6}$ At least (see later) four components (E, Z, DHP and P) are thus generally found in an irradiated solution of the $\mathrm{E}$ or $\mathrm{Z}$ isomers.

The ratio of the isomerization/cyclization quantum yields $\left(\phi_{\mathrm{Z} \rightarrow \mathrm{E}} \phi_{\mathrm{Z} \rightarrow \mathrm{DHP}}\right)$ depends on the $\mathrm{Z}$ structure. In the case of stilbene, the ratio in non-polar solvents is about $70: 30 \%$. However, only one third of the cyclization pathway in the excited state leads to the metastable ground state DHP (half life, $t_{1 / 2}$, of the order of 100 minutes) while the rest reverts to $\mathrm{Z}$ with the result of a $Z \rightarrow$ DHP quantum yield of about $10 \%$ in non-polar solvents. ${ }^{7-9}$ In the dark and in the absence of oxidants, or under irradiation, DHP undergoes ring opening and return to $\mathrm{Z}$, whereas in the presence of oxidants (e.g., oxygen or iodine) it converts thermally and irreversibly to the cyclic product $\mathrm{P}$ by hydrogen elimination, sometimes in competition with ring opening to $\mathrm{Z}$.

In some particular cases, more than one DHP can be present in the mixture since the initial DHP, generally in anaerobic conditions, can produce an isomeric intermediate, DHP', which does not revert to $\mathrm{Z}$ and loses (sometimes slowly in deaerated media) the hydro-

\footnotetext{
$\dagger$ Dedicated to Dr. Mirjana Eckert-Maksić on the occasion of her $70^{\text {th }}$ birthday.

* Authors to whom correspondence should be addressed. (E-mail: anna.spalletti@unipg.it; msindler@fkit.hr)
} 
gen atoms to give the final stable P-type photoproduct. The process implies an intramolecular 1,n-hydrogen shift $^{5}$ which can be base-catalysed, e.g. by a primary amine, the latter operating as the transferring agent. ${ }^{10}$

The photobehaviour of stilbene-analogues containing one or two heteroatoms, comparatively less studied with respect to stilbene, is interesting because the presence of heteroatoms can significantly affect the photoreaction mechanism. Pioneering work on compounds bearing pyridine or pentatomic groups [furan (F), thiophene $(\mathrm{T})$ and pyrrole $(\mathrm{Py})]$ has been carried out in the ' 60 s by various groups. ${ }^{11-13}$ More recent papers deal particularly with the solvent and oxygen dependent photocyclization pathways and with the competitive photoreactions induced by $1, n$-hydrogen shifts in stilbene-like compounds containing the pyridyl group ${ }^{14}$ or five-membered heterocycles. ${ }^{15-18}$ In fact, the photocyclization of styryl derivatives of $\mathrm{F}, \mathrm{T}$ and Py bearing various substituents at the phenyl ring and/or in specific media was described to be accompanied by high chemical yields of competitive photoproducts implying a novel skeletal rearrangement. In a study of the parasubstituted $Z$-styrylfuran in dichloromethane ${ }^{15}$ a good chemical yield of 5-(3-oxo-1-butenylbenzo[b]furan was found in the absence of oxygen and explained by the fact that the furan oxygen might cause an higher acidity of the hydrogen atom that initiates a $[1,9]$-hydrogen shift, followed by ring opening and rearrangement to this new final product. Similar results were obtained for styrylthiophene and styrylpyrrole analogues. ${ }^{16,17} \mathrm{~A}$ detailed flash photolysis study of 4'-benzyl-5-styrylfuran in DCM allowed to detect the spectra of all the intermediates and again reported the abundant competitive formation of the butadienyl derivative in the absence of oxygen. ${ }^{18}$ Another group studied the dye-sensitized photooxidation of 3-styrylthiophene and described the prevalent formation of the P-type product accompanied by formation of various oxidation products via a superoxide radical anion pathway. ${ }^{19}$

The different $\pi, \pi^{*}$ excited state distribution and different sensitivity to oxidants relative to stilbene makes pyrrole and other $\pi$-excessive five-memberedring derivatives easily decomposable unless a protective electron-acceptor substituent is present. ${ }^{20}$

The photobehaviour of stilbene analogues where a phenyl group is replaced by a pentatomic group with two heteroatoms, such as oxazole (Ox), thiazole (Th) and imidazole ( $\mathrm{Im})$ derivatives has been little investigated. A paper on styrylbenzothiazole describes the ring size effects on its photochemistry and photophysics. ${ }^{21}$ An extensive study of 5-styryloxazole (5-StOx) and analogous compounds where the other heteroaryl group was furan or thiophene, was recently carried out by some of us in the presence of iodine until full conversion. Similar good chemical yields (50-60\%) were obtained for the first three cyclized products (naphthoxazole, furobenzoxazole and thienobenzoxazole). ${ }^{22}$

The present paper deals with the quantum yields of the competitive photoreactions (isomerization/cyclization) of 5-StOx and its $p$-methoxy derivative, 5-(4-methoxystyryl)oxazole ( $p$-OMe-5-StOx). Our main aim is the study of the photoisomerization and photodehydrocyclization under mild conditions, starting from the $\mathrm{E}$ isomers, to get information on the quantum yields and photoreaction mechanism in the primary steps of irradiation. The results obtained will be briefly compared with those reported for stilbene and with the scarce quantitative information available for fivemembered heteroaryl analogues.

\section{EXPERIMENTAL}

The compounds investigated (Scheme 1) were prepared by the Van Leusen reaction from corresponding cinnamaldehydes and TosMIC reagent in the Zagreb laboratory as described previously. ${ }^{22}$ The solvents used were cyclohexane $(\mathrm{CH})$ and acetonitrile $(\mathrm{MeCN})$ from SigmaAldrich. The use of combined HPLC and UV spectrometric techniques allowed the separate spectra of the components, produced under irradiation of solutions of the starting $\mathrm{E}$ isomers, to be obtained.

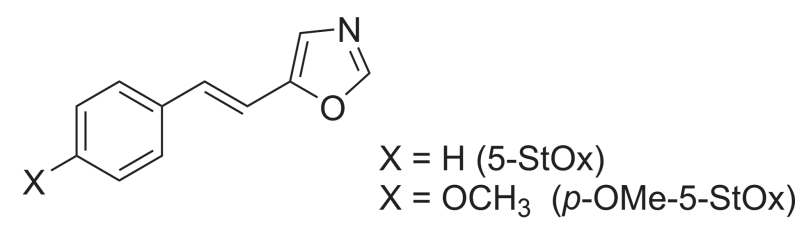

Scheme 1. Investigated compounds.

A Perkin-Elmer Lambda 800 spectrophotometer was used for the absorption measurements. The fluorescence spectra were measured with a Spex Fluorolog-2 F112AI spectrofluorimeter using dilute solutions (absorbance $<0.1$ at the excitation wavelength, $\left.\lambda_{\text {exc }}\right)$. The fluorescence quantum yield $\left(\phi_{\mathrm{F}}\right.$, uncertainty $\left.\pm 15 \%\right)$ was determined at $\lambda_{\text {exc }}$ corresponding to the maximum of the first absorption band $\left(\lambda_{\max }\right)$ using $\alpha$-NPD $\left(\phi_{\mathrm{F}}=\right.$ $0.70)^{23}$ in de-aerated $\mathrm{CH}$ as fluorimetric standard.

For photochemical measurements (potassium ferrioxalate in water was used as actinometer) a $150 \mathrm{~W}$ high pressure xenon lamp coupled with a monochromator was used. The photoreaction (solute concentrations $\sim 10^{-4} \mathrm{~mol} \mathrm{dm}^{-3}$ ) was monitored by HPLC using a Waters apparatus equipped with analytical Symmetry C18 $(4.6 \times 250 \mathrm{~mm} ; 5 \mu \mathrm{m})$ and Prontosil 200-3-C30 (4.6 $\times$ $250 \mathrm{~mm} ; 3 \mu \mathrm{m}$ ) columns and UV diode-array detector.

The procedure used to measure the photoreaction quantum yields was as follows. Diluted solutions of the 
E isomers in de-aerated (by bubbling nitrogen or argon) cyclohexane were irradiated up to a maximum conversion percentage of $10 \%$ to minimize competition of the back photoreactions. To follow the photoreactions of the $\mathrm{Z}$ isomers (not available as pure separated compounds) irradiation was initially performed at $313 \mathrm{~nm}$ (5-StOx) and $333 \mathrm{~nm}\left(\mathrm{OCH}_{3}\right.$-derivative) to reach a photostationary state enriched in the $\mathrm{Z}$ isomer (in the presence of a side source of visible light to destroy DHP and avoid P formation at this stage) and then at $254 \mathrm{~nm}$ where the absorption of the latter largely prevails. Samples of the irradiated solutions, analyzed immediately after the removal of the irradiating source by combined HPLC and spectrophotometric techniques, generally contain four components: E, Z, DHP and small amounts of P-type dehydrogenated arene. The air-equilibrated solutions were then left overnight in the dark to allow the decay of the DHP intermediates to go to completion. The residual components were separated by HPLC (using acetonitrile as eluent, a small addition of water being necessary in some cases to better separate the chromatographic peaks) and identified spectrophotometrically.

In the case of the $p$-OMe derivative, where the possible conformers of the $\mathrm{Z}$ isomer have comparable abundances (see Results and discussion), the quantum yields of $\mathrm{E}$ and $\mathrm{P}$ formation $\left(\phi_{\mathrm{Z} \rightarrow \mathrm{E}}\right.$ and $\left.\phi_{\mathrm{Z} \rightarrow \mathrm{P}}\right)$ measured at $\lambda_{\text {exc }}=254 \mathrm{~nm}$, could be affected by different absorption spectra of the two conformers. In this case, the reported quantum yields are to be considered mean values for the conformer mixture.

The thermal stability of DHPs, usually expressed by the half-lives $\left(t_{1 / 2}\right)$ at room temperature, was measured by following spectrophotometrically the disappearance of the visible absorption band in both aerated and de-aerated (by bubbling nitrogen or argon) $\mathrm{CH}$. The quantum yields reported in the Tables are generally the averages of three independent experiments with mean deviations of $c a .5 \%$ for $\phi_{\mathrm{E} \rightarrow \mathrm{Z}}$. Higher uncertainties $(10-15 \%)$ were found in the more delicate measurements of the photoreactions starting from the $\mathrm{Z}$ isomer $\left(\phi_{\mathrm{Z} \rightarrow \mathrm{E}}\right.$ and $\left.\phi_{\mathrm{Z} \rightarrow \mathrm{P}}\right)$, particularly considering the very small cyclization yield.

Quantum-mechanical calculations were carried out using the HyperChem computational package (version 7.5).

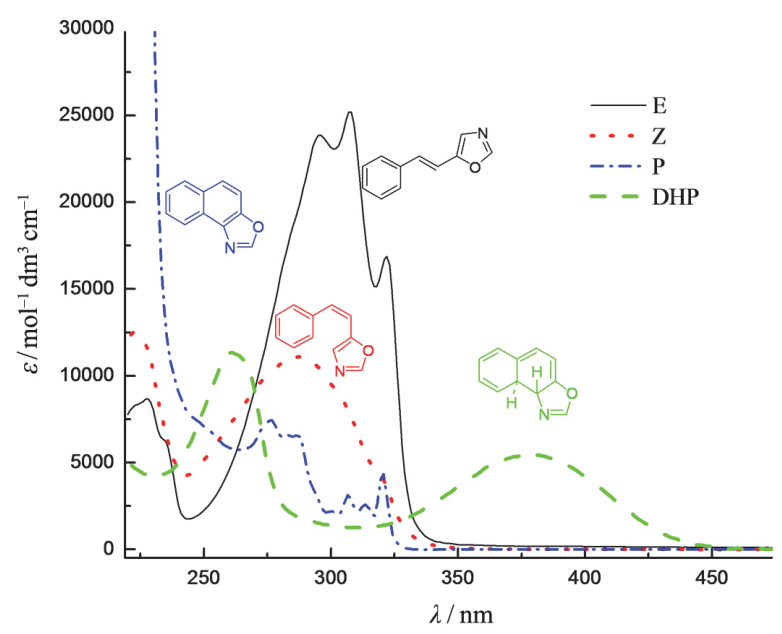

Figure 1. Absorption spectra of the two geometrical isomers (E and $\mathrm{Z}$ ) of 5-StOx in $\mathrm{CH}$ together with the DHP intermediate and its oxidation P-type product.

Total energies and dipole moments were obtained for geometries optimized by HF ab initio method (3-21-G level). The computed transition energies and oscillator strengths were obtained by ZINDO/S at the optimized geometries, the configuration interaction including 400 $(20 \times 20)$ single excited configurations.

\section{RESULTS AND DISCUSSION}

\section{5-styryloxazole (5-StOx)}

Figure 1 shows the absorption spectra of $E$-5-StOx and its photoproducts in $\mathrm{CH}$ while Table 1 collects the main spectral properties of the $\mathrm{E}$ and $\mathrm{Z}$ isomer, respectively, together with those of the $p$-OMe derivative, in $\mathrm{CH}$ and $\mathrm{MeCN}$. The spectra of the $\mathrm{Z}$ isomer and the DHP-type intermediate were obtained by normalization at the $\mathrm{Z} / \mathrm{E}$ and DHP/P isosbestic points, respectively. The spectrum of the final P-type product in $\mathrm{CH}$ was considered to have the same absorption coefficient as that previously reported in $\mathrm{EtOH}^{22}$ While the $\mathrm{P}$ spectrum displays a slightly structured shape, the DHP spectrum shows a typical bellshaped band shifted to the red at $380 \mathrm{~nm}$, characteristic of an increased conjugation in the closed form. ${ }^{4,5}$

The presence of the $\mathrm{N}$ heteroatom (not implied in the aromatic sextet) as a new site of basicity decreases

Table 1. Absorption and fluorescence maxima of the E isomer of the compounds investigated in two solvents

\begin{tabular}{ccccc}
\hline Compound & Solvent & $\lambda_{\mathrm{ab}} / \mathrm{nm}^{(\mathrm{a})}$ & $\varepsilon_{\mathrm{ab}} \mathrm{max}^{\mathrm{max}} / \mathrm{mol}^{-1} \mathrm{dm}^{3} \mathrm{~cm}^{-1}$ & $\lambda_{\mathrm{fl}} / \mathrm{nm}^{(\mathrm{a})}$ \\
\hline \multirow{2}{*}{ 5-StOx } & $\mathrm{CH}$ & $296, \underline{308}, 322$ & 25190 & $328, \underline{343}, 359$ \\
& $\mathrm{MeCN}$ & $293, \underline{304}, 318$ & 28580 & $344, \underline{361}, 378$ \\
\multirow{2}{*}{$p$-OMe-5-StOx } & $\mathrm{CH}$ & $306, \underline{319}, 333^{\mathrm{sh}}$ & 30830 & 32270 \\
\hline
\end{tabular}

\footnotetext{
(a) The main maximum is underlined; sh means shoulder.
} 
the $\pi$-rich character of the oxazole ring with respect to the furan and pyrrole analogues, as also indicated by its higher ionization potential $(9.83 \mathrm{eV}$ vs. 8.88 and 8.90 $\mathrm{eV}$ for $\mathrm{F}$ and $\mathrm{Py}$, respectively). ${ }^{24}$ This effect bestows on this azole moiety a weaker electron donor character and leads to an electron deficiency on the $\mathrm{C}$ atoms of the ring. It has to be noted that the decrease of the electron density at the 4-C (the reactive position of the cyclization reaction) can affect the yield of the latter photoreaction, see below).

The donor properties of the Ox ring are expected to give some charge transfer (CT) character to the $\mathrm{S}_{0} \rightarrow \mathrm{S}_{1}$ transition even if the spectral behaviour detected in $\mathrm{MeCN}$ (higher polarity but lower polarization) is very similar to that in $\mathrm{CH}$ with maxima only slightly shifted towards the blue. The computed dipole moment of the ground state $\left(\mu_{\mathrm{g}}=2.42\right.$ and $1.82 \mathrm{D}$ for the $\mathrm{E}$ and $\mathrm{Z}$ isomer, respectively, see below, Table 2) reveals a slightly unsymmetrical charge distribution.

Figure 2 shows the normalized absorption and fluorescence emission/excitation spectra of E-5-StOx in $\mathrm{CH}$. The spectra are almost specular and slightly structured with an absorption band displaying a main peak at $308 \mathrm{~nm}$ in $\mathrm{CH}$ and two side peaks with vibrational progression typical of stilbenoid compounds. The emission spectrum, showing a Stokes shift of $3420 \mathrm{~cm}^{-1}$, does not

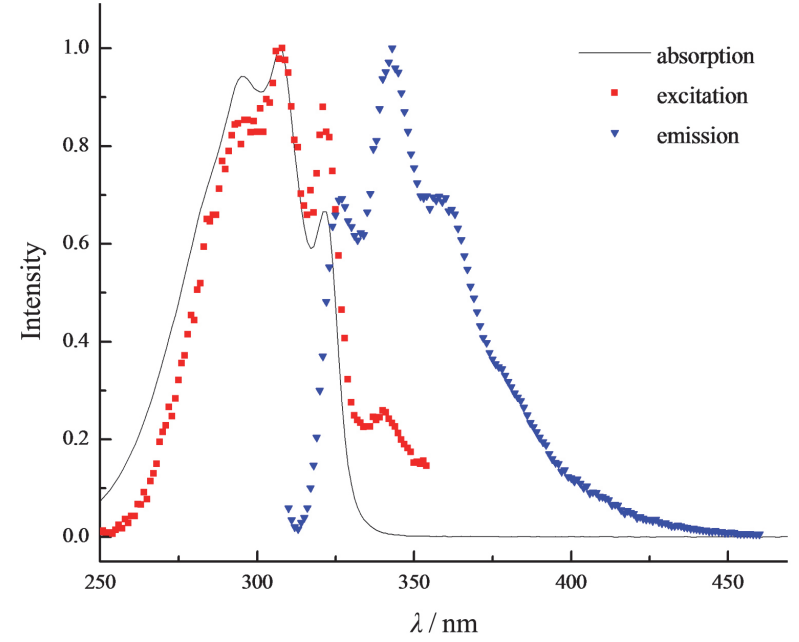

Figure 2. Normalized absorption, fluorescence (emission and excitation) spectra of $E-5-\mathrm{StOx}$ in $\mathrm{CH}$.

depend on the excitation wavelength $\left(\lambda_{\mathrm{exc}}\right)$ while the excitation spectrum shows a non-negligible change in the relative intensities of the two side peaks that does not exclude a small effect of conformational equilibria. ${ }^{25,26}$

Theoretical calculations were then carried out on the formation enthalpy to evaluate the presence of conformational equilibria in our solutions (Scheme 2).

Table 2. Computed dipole moment $(\mu / \mathrm{D})$ and total energy $\left(E_{\text {ТОТ }} / \mathrm{kcal} \mathrm{mol}^{-1}\right)$, derived relative abundance $(\%)$ at room temperature, transition energy $(\lambda / \mathrm{nm})$ and oscillator strength $(f)$ of the conformers of the $\mathrm{E}$ and $\mathrm{Z}$ isomers of 5-StOx

\begin{tabular}{|c|c|c|c|c|c|c|}
\hline rotamer & $\mu / \mathrm{D}$ & $E_{\mathrm{TOT}} / \mathrm{kcal} \mathrm{mol}^{-1}$ & $\%$ (at $293 \mathrm{~K}$ ) & $\lambda / \mathrm{nm}$ & $f$ & \\
\hline$E-5-\mathrm{StOx}(\mathrm{A})$ & 2.42 & -343864.719 & 1 & $\begin{array}{l}301 \\
254 \\
217 \\
198 \\
190\end{array}$ & $\begin{array}{l}1.06 \\
0.07 \\
0.31 \\
0.07 \\
0.39\end{array}$ & $\begin{array}{c}\mathrm{S}_{1} \\
\mathrm{~S}_{3} \\
\mathrm{~S}_{7} \\
\mathrm{~S}_{9} \\
\mathrm{~S}_{11}\end{array}$ \\
\hline$E-5-\mathrm{StOx}(\mathrm{B})$ & 2.42 & -343867.188 & 99 & $\begin{array}{l}312 \\
259 \\
218 \\
199\end{array}$ & $\begin{array}{l}0.98 \\
0.18 \\
0.40 \\
0.11\end{array}$ & $\begin{array}{l}\mathrm{S}_{1} \\
\mathrm{~S}_{3} \\
\mathrm{~S}_{7} \\
\mathrm{~S}_{9}\end{array}$ \\
\hline$Z$-5-StOx (A) & 1.82 & -343862.469 & 97 & $\begin{array}{l}282 \\
237 \\
213 \\
196\end{array}$ & $\begin{array}{l}0.50 \\
0.09 \\
0.09 \\
0.80\end{array}$ & $\begin{array}{l}\mathrm{S}_{1} \\
\mathrm{~S}_{8} \\
\mathrm{~S}_{11} \\
\mathrm{~S}_{12}\end{array}$ \\
\hline$Z-5-S t O x(B)$ & 1.84 & -343860.500 & 3 & $\begin{array}{l}286 \\
232 \\
192 \\
189\end{array}$ & $\begin{array}{l}0.34 \\
0.52 \\
0.77 \\
0.91\end{array}$ & $\begin{array}{c}\mathrm{S}_{1} \\
\mathrm{~S}_{5} \\
\mathrm{~S}_{12} \\
\mathrm{~S}_{13}\end{array}$ \\
\hline
\end{tabular}

Table 3. Absorption properties of the $\mathrm{Z}$ and DHP photoproducts and DHP lifetime for the two compounds investigated in $\mathrm{CH}$

\begin{tabular}{ccccc}
\hline Compound & $\lambda \mathrm{Z} / \mathrm{nm}^{(\mathrm{a})}$ & $\varepsilon Z^{\max } / \mathrm{mol}^{-1} \mathrm{dm}^{3} \mathrm{~cm}^{-1}$ & $\lambda_{\mathrm{DHP}} / \mathrm{nm}^{\text {(a) }}$ & $\tau_{\mathrm{DHP}} / \mathrm{min}$ \\
\hline 5-StOx & $\underline{288}, 306^{\text {sh }}, 320^{\text {sh }}$ & 11100 & 378 & 200 \\
$p$-OMe-5-StOx & $\underline{297}, 318^{\text {sh }}, 330^{\text {sh }}$ & 15520 & 378 & 100 \\
\hline
\end{tabular}

(a) The main maximum is underlined; sh means shoulder. 
Table 2 shows the computed spectral properties and

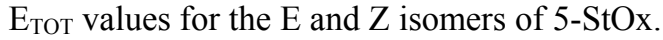<smiles>CCc1cnco1</smiles>

$s$-cis rotamer (A)

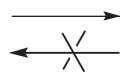

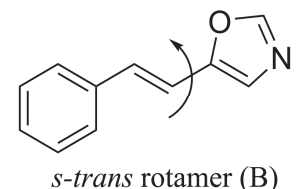

s-trans rotamer (B)
Scheme 2. Conformational isomers of E-5-StOx.

The results for 5-StOx showed a good agreement between the experimental and computed absorption maxima while the $E_{\text {Tот }}$ values indicated that one conformer is expected to be largely prevalent for both geometrical isomers, the second one being practically negligible ( $1 \%$ for $\mathrm{E}$ and $3 \%$ for $\mathrm{Z}$ ).

The quantum yields of the radiative and reactive processes of 5-StOx are shown in Table 4 together with those of its substituted analogue. The fluorescence yield of 5-StOx was found to be $\phi_{\mathrm{F}}=0.072$ in deaerated $\mathrm{CH}$ while the corresponding lifetime was found to be shorter than the resolution time of our apparatus ( $0.5 \mathrm{~ns})$. An increase in the $\phi_{\mathrm{F}}$ value which seemed to be at the limits of the experimental uncertainty was observed using $\lambda_{\text {exc }}$ longer than the absorption maximum.

As tested in a previous work, ${ }^{27}$ the combined use of HPLC and spectrophotometric techniques was very useful for the separation of the components of the photoreaction mixture thus allowing the evaluation of the isomerization/cyclization competition and the measurement of the spectra and decay kinetics of the DHP-type intermediate. Our experiments in $\mathrm{CH}$ were carried out in different steps. Initial irradiation at $313 \mathrm{~nm}$ was used to measure the $\mathrm{E} \rightarrow \mathrm{Z}$ photoisomerization quantum yield. Prolonged irradiation produced a $\mathrm{E} / \mathrm{Z}$ photostationary state. In order to enrich the mixture in the $\mathrm{Z}$ isomer the solution was subjected to a parallel irradiation with light of $\lambda_{\mathrm{exc}}>350 \mathrm{~nm}$ to avoid the ring closure. The mixture thus obtained $(\cong 80 \% \mathrm{Z})$ underwent a further irradiation step at $254 \mathrm{~nm}$ to follow the competitive $Z \rightarrow E$ and $\mathrm{Z} \rightarrow$ DHP photoreactions. Left overnight in aerated conditions, the mixture showed a decrease of DHP and an increase in P. Otherwise, when irradiated at $380 \mathrm{~nm}$ with light absorbed by DHP only, the solution showed an increase (from 20 to $80 \%$ ) in the $Z$ component confirming the photochemical DHP $\rightarrow \mathrm{Z}$ back ring opening, as well known for the corresponding hydrocarbon. ${ }^{4,5}$

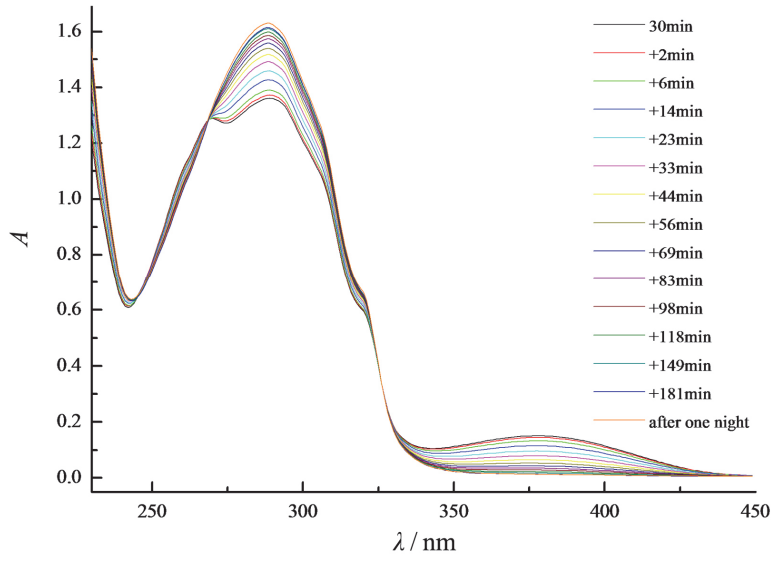

Figure 3. Spectral evolvement under irradiation of 5-StOx in $\mathrm{CH}$ : this solution was previously irradiated for $30 \mathrm{~min}$ at $\lambda=313$ $\mathrm{nm}$ to produce DHP and then further irradiated at $\lambda=380 \mathrm{~nm}$.

As a matter of fact, accurate analysis of the reaction mixture evidenced the formation of a very small amount of a fifth component X. The possibility that such intermediate could be produced through a $1, n$-hydrogen shift was explored in details but with negative result. In fact, the HPLC analysis showed that X was induced by the polar eluent mixture (MeCN containing small amounts of water) while mass-chromatographic analysis indicated that it contained oxygen and thus was assignable to a negligible side product of oxidation favoured by the eluent in aerated conditions.

Going to the photoreaction quantum yields summarized in Table 4 for the two compounds investigated, the $\mathrm{E} \rightarrow \mathrm{Z}$ photoisomerization of 5-StOx, was found to have a quantum yield $\phi_{\mathrm{E} \rightarrow Z}=0.57$, a value close to that of stilbene $(\sim 0.50) .{ }^{1,2}$ The high value of the reaction quantum yield (even higher than the value of 0.5 expected by the diabatic mechanism ${ }^{2}$ ), accompanied by the small emission yield, indicates that decay by internal conversion (IC) can be excluded for this compound and probably the photoreaction occurs in the singlet manifold through a diabatic mechanism. It should be noted that the few quantitative data of relaxation quantum yields available in the literature for $E$-styryl-substituted five-membered heteroaryl units $\left(\mathrm{T},{ }^{28,29} \mathrm{Py},{ }^{30}\right.$ indole $\left.^{30}\right)$ indicate that these structures are characterized by a similar photobehaviour, namely efficient isomerization and weak fluorescence. Only for 2-styrylbenzothiazole a much less efficient reactive pathway was reported with $\mathrm{E} \rightarrow \mathrm{Z}$ quantum yields of the order of $10 \%$, probably

Table 4. Fluorescence and photoreaction quantum yields of the compounds investigated in $\mathrm{CH}$

\begin{tabular}{ccccc}
\hline Compound & $\phi \mathrm{F}$ & $\phi \mathrm{E} \rightarrow \mathrm{Z}$ & $\phi \mathrm{Z} \rightarrow \mathrm{E}$ & $\phi \mathrm{Z} \rightarrow \mathrm{P}$ \\
\hline 5-StOx & $0.072 \pm 0.009$ & $0.57 \pm 0.01$ & $0.15 \pm 0.01$ & $0.013 \pm 0.0015$ \\
$p$-OMe-5-StOx & $0.026 \pm 0.003$ & $0.41 \pm 0.03$ & $0.24 \pm 0.02$ & $0.027 \pm 0.003$ \\
\hline
\end{tabular}


due to a sizable contribution of ICT processes competitive with isomerization. ${ }^{21}$

Experiments carried out with increasing oxygen concentrations showed an expected decrease in the DHP lifetime: from 200 to 150 and 90 min on going from deaerated (by bubbling nitrogen) to aerated and oxygensaturated $\mathrm{CH}$ solution, respectively. A similar value (96 min) was reported for stilbene in a non-polar $2: 1$ mixture of methylcyclohehane/isohexane. ${ }^{4}$

The quantum yield of the competitive $\mathrm{Z} \rightarrow \mathrm{E}$ and $\mathrm{Z} \rightarrow \mathrm{P}$ in Table 4 was preferably investigated using $\lambda_{\mathrm{exc}}$ of $254 \mathrm{~nm}$ where $Z$ is the main absorber. The values thus obtained were $\phi_{\mathrm{Z} \rightarrow \mathrm{E}}=0.15$ and $\phi_{\mathrm{Z} \rightarrow \mathrm{P}}=0.013$. The isomerization yield is rather smaller than that reported for stilbene (0.35) while the cyclization yield (to give naphtho[1,2-d]oxazole, NOx) is almost one order of magnitude smaller than the value of 0.10 reported for stilbene. $^{7-9}$ As said above, the small cyclization yield based on the DHP interconversion to $\mathrm{P}$ in the presence of oxidant (left overnight in the dark) has a higher uncertainty with respect to the yields of the other photoreactions.

\section{5-(4-methoxystyryl)oxazole ( $p$-OMe-5-StOx)}

The spectral properties of this compound bearing a donor para-substituent are rather similar to those of 5-StOx. They showed a bathochromic shift of $12 \mathrm{~nm}$ and an increase in the absorption coefficient of 22 and $13 \%$ in non-polar and polar solvent, respectively, with respect to the parent compound.

Table 5 shows the computed spectral properties

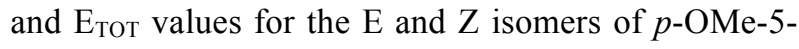
$\mathrm{StOx}$. Even for this compound the $E_{\mathrm{TO}}$ values indicated that one conformer (A) largely prevails for the E isomer while a mixture of two conformers is foreseen for the $\mathrm{Z}$ isomer.
It is known that methoxy-substitution at the ortho and para positions leads to a decrease in the ionization potential (favouring the donor properties) and in the energy gap (increase of the HOMO energy and red shift of the spectrum) of the transition. The higher computed dipole moment of the $\mathrm{E}$ isomer $(\mu=3.38 \mathrm{D})$ reflects the combined effect of the donor properties of the two side groups. However, the expected increased contribution of ICT was not verified, the effect of the solvent polarity on the absorption spectrum (small hypsochromic and auxochromic shifts) being similar to that measured for the unsubstituted compound. A most important effect was found on the emission properties. A modest but significant reduction in both $\phi_{\mathrm{F}}$ and $\phi_{\mathrm{E} \rightarrow \mathrm{Z}}$ values $(0.026$ and 0.41 , respectively) was found for the $p$-OMe derivative in $\mathrm{CH}$ with respect to the unsubstituted compound. Even in this case, the $\phi_{\mathrm{F}}$ value showed a slight increase using longer $\lambda_{\text {exc }}$ after the absorption maximum. The possible contribution of ISC to the triplet state was not investigated but a moderate triplet production cannot be excluded. It was interesting to observe that the fluorescence emission, weak in $\mathrm{CH}$, becomes undetectable in $\mathrm{MeCN}$.

The photobehaviour of Z-p-OMe-5-StOx was studied with the procedure used for the parent compound and gave similar results. The spectrum of the DHP intermediate in $\mathrm{CH}$ was clearly observable with $\lambda_{\max }=380 \mathrm{~nm}$ in $\mathrm{CH}$. The lifetime measured in aerated solution in the first two hours of its bleaching was about 100 min with a tendency to increase after further decay, probably because of the consumption of the oxidant. The absorption spectrum of the final cyclized product (8-methoxynaphtho[1,2-d]oxazole, 8-OMe-NOx) in $\mathrm{MeCN}$ was in good agreement with that previously reported in $\mathrm{EtOH}^{22}$ The $\phi_{Z \rightarrow E}(0.24)$ and $\phi_{Z \rightarrow P}(0.027)$ were found almost doubled with respect to the unsubstituted compound.

Table 5. Computed dipole moment $(\mu / \mathrm{D})$ and total energy $\left(E_{\mathrm{TOT}} / \mathrm{kcal} \mathrm{mol}^{-1}\right)$, derived relative abundance $(\%)$ and electronic spectra [transition energy $(\lambda / \mathrm{nm})$ and oscillator strength $(f)$ ] of the conformers of the $\mathrm{E}$ and $\mathrm{Z}$ isomers of $p$-OMe-5-StOx

\begin{tabular}{|c|c|c|c|c|c|c|}
\hline rotamer & $\mu / \mathrm{D}$ & $E_{\mathrm{TOT}} / \mathrm{kcal} \mathrm{mol}^{-1}$ & $\%($ at $T=293 \mathrm{~K})$ & $\lambda / \mathrm{nm}$ & $f$ & \\
\hline$E-p-\mathrm{MeO}-5-\mathrm{StOx}(\mathrm{A})$ & 3.38 & -414934.625 & 99 & $\begin{array}{l}315 \\
260 \\
216\end{array}$ & $\begin{array}{l}1.08 \\
0.14 \\
0.40\end{array}$ & $\begin{array}{l}\mathrm{S}_{1} \\
\mathrm{~S}_{3} \\
\mathrm{~S}_{7}\end{array}$ \\
\hline$E-p-\mathrm{MeO}-5-\mathrm{StOx}(\mathrm{B})$ & 3.63 & -414932.156 & 1 & & & \\
\hline$Z-p-\mathrm{MeO}-5-\mathrm{StOx}(\mathrm{A})$ & 1.73 & -414929.062 & 26 & $\begin{array}{l}310 \\
257 \\
225 \\
217 \\
216\end{array}$ & $\begin{array}{l}0.63 \\
0.38 \\
0.08 \\
0.26 \\
0.08\end{array}$ & $\begin{array}{l}\mathrm{S}_{1} \\
\mathrm{~S}_{3} \\
\mathrm{~S}_{6} \\
\mathrm{~S}_{7} \\
\mathrm{~S}_{8}\end{array}$ \\
\hline$Z-p-\mathrm{MeO}-5-\mathrm{StOx}(\mathrm{B})$ & 2.19 & -414929.660 & 74 & $\begin{array}{l}285 \\
228 \\
220 \\
203 \\
194\end{array}$ & $\begin{array}{l}0.50 \\
0.19 \\
0.11 \\
0.21 \\
0.40\end{array}$ & $\begin{array}{l}\mathrm{S}_{1} \\
\mathrm{~S}_{6} \\
\mathrm{~S}_{8} \\
\mathrm{~S}_{10} \\
\mathrm{~S}_{13}\end{array}$ \\
\hline
\end{tabular}




\section{CONCLUSIONS}

The study of 5-StOx and p-OMe-5-StOx under mild conditions showed a photobehaviour similar to that of stilbene, at least regarding the deactivation of the $\mathrm{E}$ isomer. Taken together, the yields of the abundant isomerisation and relatively weak fluorescence account for all the quanta absorbed. Both yields are slightly lower for the $p$-OMe-derivative where a weak contribution of internal conversion cannot be excluded. The situation is different for the $\mathrm{Z}$ isomer since the two competitive photoreactions (back isomerization to $\mathrm{E}$ and cyclization) are characterized by lower yields compared to stilbene, particularly for the unsubstituted compound where the cyclization yield decreases by almost an order of magnitude with respect to stilbene. This effect could be due to the reduced electron density (with respect to the analogous five-membered rings with only one heteroatom, F and Py), caused by the presence of the basic $\mathrm{N}$ heteroatom, at the reaction center, namely on the carbon atom implied in the ring closing reaction. A general quantitative study of the photobehaviour of these stilbene analogues bearing different fivemembered heteroaryl groups would be necessary to better understand the structure effect on the relaxation properties and the possible interest for applications. We plan to extend this research line on structurallymodified analogous compounds where the presence of pentatomic heteroaryl group will probably induce larger effects. The continuation of this work in different directions has the aim of investigating the effects of: i) positional isomers bearing the styryl group in different positions (2- and 4-StOx); ii) fused-ring bicyclic benzologs (styrylbenzoxazoles), and iii) diarylethenes containing two heteroaryl groups with the same (symmetric) or different (asymmetric) electron donor/acceptor properties. The latter are expected to be of specific interest since the occurring of intramolecular CT processes in the excited state should strongly affect the radiative and reactive relaxation leading to properties of potential application.

Acknowledgements. The authors recognize the financial support by MIUR (Ministero dell'Università e della Ricerca, Rome, Italy) and the University of Perugia (PRIN 2010-2011 n. 2010FM738P) as well as the Ministry of Science, Education and Sports of the Republic of Croatia (grant nos. 1250982933-2926). The authors thank also Mr. D. Pannacci for his technical assistance in HPLC measurements.

\section{REFERENCES}

1. D. H. Waldeck, Chem. Rev. 91 (1991) 415-436 and references therein.
2. J. Saltiel and Y.-P. Sun, in H. Dürr, H. Bouas-Laurent (Eds.) Photochromism: Molecules and Systems, Elsevier, Amsterdam, 1990, pp. 64-162, and references therein.

3. G. Bartocci, U. Mazzucato, and A. Spalletti, Trends Phys. Chem. 12 (2007) 1-36.

4. K. A. Muszkat, Top. Curr. Chem. 88 (1980) 91-143 and references cited therein.

5. F. B. Mallory and C. W. Mallory, in Organic Reactions, John Wiley and Sons: New York, 1984, Vol. 30, pp 1-456.

6. W. H. Laarhoven, in H. Dürr, H. Bouas-Laurent (Eds.), Photochromism, Molecules and Systems, Elsevier, Amsterdam, 1990, chapter 7, pp. 270-313 and references therein.

7. K. A. Muszkat and E. Fischer, J. Chem. Soc. B (1967) 662-678 and references therein.

8. T. Wismontski-Knittel, G. Fischer, and E. Fischer, J. Chem. Soc. Perkin Trans. II (1974) 1930-1940.

9. H. Petek, K. Yoshihara, Y. Fujiwara, Z. Lin, J. H. Penn, and J. H. Frederick, J. Phys. Chem. 94 (1990) 7539-7543 and references cited therein.

10. J. B. M. Somers, A. Couture, A. Lablache-Combier, and W. H. Laarhoven, J. Am. Chem. Soc. 107 (1985) 1387-1394 and references therein.

11. C. E. Loader and C. J. Timmons, J. Chem. Soc. (C) (1967) $1677-$ 1681.

12. W. Carruthers and H. N. M. Stewart, J. Chem. Soc. (1965) 62216227.

13. V. H. Rawai, R. J. Jones, and M. P. Cava, Tetrahedron Lett. 26 (1985) 2423-2426 and references therein.

14. F. D. Lewis, T. L. Kurth, R. S. Kalgutkar, and J.-S. Yang, J. Am. Chem. Soc. 123 (2001) 3878-3884.

15. T.-I. Ho, J.-Y. Wu, and S.-L. Wang, Angew. Chem. Int. Ed. 38 (1999) 2558-2560.

16. J.-Y. Wu, J.-H. Ho, S.-M. Shih, T.-L. Hsieh, and T.-I. Ho, Org. Lett. 1 (1999) 1039-1041.

17. J.-H. Ho and T.-I. Ho, Tetrahedron Lett. 44 (2003) 4669-4672.

18. S. Samori, M. Hara, T.-I. Ho, S. Tojo, K. Kawai, M. Endo, M. Fujitsuka, and T. Majima, J. Org. Chem. 70 (2005) 2708-2712.

19. K. Song, L.-Z. Wu, C.-H. Yang, and C.-H. Tung, Tetrahedron Lett. 41 (2000) 1951-1954.

20. B. Antelo, L. Castedo, J. Delamano, A. Gómez, C. López, and G. Toyo, J. Org. Chem. 61 (1996) 1188-1189.

21. M. K. Awad, M. M. El-Hendawy, T. A. Fared, S. E. H. Etaiw, and N. J. English, Photochem. Photobiol. Sci. 12 (2013) 12201231.

22. I. Šagud, F. Faraguna, Ž. Marinić, and M. Šindler-Kulyk, J. Org. Chem. 76 (2011) 2904-2908.

23. G. Bartocci, F. Masetti, U. Mazzucato, A. Spalletti, I. Baraldi and F. Momicchioli, J. Phys. Chem. 91 (1987) 4733-4743.

24. M. Montalti, A. Credi, L. Prodi, and M. T. Gandolfi, Handbook of Photochemistry, 3. Ed., CRC Press, 2006, pp. 500-501.

25. U. Mazzucato and F. Momicchioli, Chem. Rev. 91 (1991) 1679 1719.

26. G. Bartocci, A. Spalletti, and U. Mazzucato, in Waluk, J. (Ed.), Conformational Analysis of Molecules in Excited States, Wiley$\mathrm{VCH}$, New York, 2000, ch. 5 and references therein.

27. U. Mazzucato and A. Spalletti, J. Phys. Chem. A 113 (2009) 14521-14529 and references therein.

28. L. L. Costanzo, S. Pistarà, G. Condorelli, and G. Scarlata, J. Photochem. 7 (1977) 297-304.

29. P. Gajdek, R. S. Becker, F. Elisei, U. Mazzucato, and A. Spalletti, J. Photochem. Photobiol. A: Chem. 100 (1996) 57-64.

30. G. Galiazzo, G. Gennari, and P. Bortolus, Gazz. Chim. It. 121 (1991) 67-71. 